\title{
Monitoring the thermally induced structural transitions of collagen by use of second-harmonic generation microscopy
}

\author{
Sung-Jan Lin \\ Institute of Biomedical Engineering, College of Medicine and College of Engineering, \\ National Taiwan University, Taipei 100, Taiwan, \\ and Department of Dermatology, National Taiwan University Hospital, Taipei 100, Taiwan
}

Chih-Yuan Hsiao

Institute of Electro Optics, Department of Electrical Engineering, National Taiwan University, Taipei 100, Taiwan

Yen Sun and Wen Lo

Department of Physics, National Taiwan University, Taipei 106, Taiwan

Wei-Chou Lin

Department of Pathology, National Taiwan University Hospital, Taipei 100, Taiwan

Gwo-Jen Jan

Institute of Electro Optics, Department of Electrical Engineering, National Taiwan University, Taipei 100, Taiwan

Shiou-Hwa Jee

Department of Dermatology, National Taiwan University Hospital, Taipei 100, Taiwan, and Department of Dermatology, National Taiwan University College of Medicine, Taipei 100, Taiwan

\author{
Chen-Yuan Dong \\ Department of Physics, National Taiwan University, Taipei 106, Taiwan
}

Received September 10, 2004

The thermal disruption of collagen I in rat tail tendon is investigated with second-harmonic generation (SHG) microscopy. We investigate its effects on SHG images and intensity in the temperature range $25^{\circ}-60^{\circ} \mathrm{C}$. We find that the SHG signal decreases rapidly starting at $45^{\circ} \mathrm{C}$. However, SHG imaging reveals that breakage of collagen fibers is not evident until $57^{\circ} \mathrm{C}$ and worsens with increasing temperature. At $57^{\circ} \mathrm{C}$, structures of both molten and fibrous collagen exist, and the disruption of collagen appears to be complete at $60^{\circ} \mathrm{C}$. Our results suggest that, in addition to intensity measurement, SHG imaging is necessary for monitoring details of thermally induced changes in collagen structures in biomedical applications. () 2005 Optical Society of America

OCIS codes: $190.4160,170.3880,180.0180$.

In recent years, multiphoton fluorescence microscopy has gained significant popularity in bioimaging applications. The nonlinear excitation of fluorescence photons with ultrafast, near-infrared excitation sources has important advantages in its ability to acquire enhanced axial depth discrimination images, reduced overall specimen photodamage, and increased imaging penetration depths. ${ }^{1,2}$ In addition to multiphoton fluorescence imaging, nonlinear polarization effects from a special class of biological materials also have biomedical significance. In biological structures lacking inversion symmetry a nonvanishing second-order susceptibility can contribute to a second-harmonic generation (SHG) signal given by

$$
P_{i}=\chi_{i j k}^{2} E_{j} E_{k} .
$$

A variety of biological materials, such as collagen and muscle fibers, have been shown to be effective in gen- erating second-harmonic signals. ${ }^{3,4}$ In the case of collagen, SHG imaging is of general interest since collagen is widely found in tissues such as tendon, skin, and cornea and is a major constituent of the extracellular matrix. A particularly interesting application of SHG imaging is the monitoring of thermally induced structural transitions of collagen fibers. A number of medical procedures depend on heat-induced changes in collagen fibers to achieve therapeutic results. In laser-assisted capsulorrhaphy, laser heating of collagen in the shoulder can result in fiber shrinkage and enhanced stability of the shoulder joint. ${ }^{5}$ Another procedure is conductive keratoplasty, in which current-induced heat is used to change the cornea curvature for vision correction. ${ }^{6}$ Finally, heat from a laser source can be used to tighten and rejuvenate skin. ${ }^{7}$ The thermal effect on collagen has been investigated by measurement of the second-harmonic signal. It was found that the collagen SHG signal de- 
creases at approximately $64^{\circ} \mathrm{C}$, presumably because of a structural transition in the collagen internal structure. ${ }^{8,9}$ Laser illumination has also been shown to induce thermal damage to collagen fibers. ${ }^{10}$ However, to the best of our knowledge, the correlation between the thermally induced decrease in the SHG signal and collagen structures is not completely understood. In this work we obtain the SHG images from rat tail tendon after thermal treatment in the temperature range between $25^{\circ} \mathrm{C}$ and $60^{\circ} \mathrm{C}$. We correlate the structural changes in collagen to changes in the SHG signal. An understanding of this relationship will help researchers in developing thermal procedures for biomedical applications.

The second-harmonic imaging system used in this study is a modified version of a home-built laserscanning microscopic-imaging system based on an upright microscope (E800, Nikon, Japan) described previously. ${ }^{11}$ A diode-pumped (Millennia X, SpectraPhysics, Mountain View, California), Ti:sapphire (ti-sa; Tsunami, Spectra-Physics) is used as the excitation source. The 780-nm output of the ti-sa laser is scanned in the focal plane by a galvanometer-driver $x-y$ mirror scanning system (Model 6220, Cambridge Technology, Cambridge, Massachusetts). Before entering the upright microscope, the laser is beam expanded to ensure overfilling of the objective's back aperture. For high-resolution imaging a highnumerical-aperture, oil-immersion objective (S Fluor $40 \times$, N.A. of 1.3, Nikon) was selected for SHG microscopy. To direct the expanded laser spot to the sample, a short-pass dichroic mirror (700DCSPXRUV-3p, Chroma Technology, Brattleboro, Vermont) is used to reflect the incident excitation laser source. To ensure even excitation of collagen fibers at different orientations, a $\lambda / 4$ wave plate is used to convert the linearly polarized ti-sa laser beam into one with circular polarization. The average laser power at the sample is $5.1 \mathrm{~mW}$, and the SHG signal generated at this power is found to be within the quadratic-dependence region of the SHG signal of the excitation power. The generated SHG signal is then collected in a backscattering geometry in which the dichroic mirror, a short-pass filter (E680SP, Chroma Technology), and a $390-\mathrm{nm}$ bandpass filter (HQ390/20, Chroma Technology) are used to isolate the SHG signal. The signal photons are processed by a single-photon-counting photomultiplier tube (R7400P, Hamamatsu, Japan) and a home-built discriminator.

In our study, rat tail tendon is sliced into small sections and placed in a phosphate-buffered saline buffer before being subjected to thermal baths for $10 \mathrm{~min}$. In this manner we can ensure rapid and uniform heating and cooling of the tendon specimens upon placing them into and removing them from the thermal bath. The temperature range between $25^{\circ} \mathrm{C}$ and $60^{\circ} \mathrm{C}$ is chosen for thermal treatment of the specimens. At the end of the 10-min heating cycle the tendon specimen is removed, mounted on a glass slide, and covered with a No. 1.5 cover glass for viewing. We acquire second-harmonic images of the tendon treated at different temperatures. To gain a thor- ough understanding of the effects of heating on collagen, a large-area scan of each collagen specimen composed of a $6 \times 6$ array of neighboring SHG images is acquired and assembled. The average SHG signal per pixel is computed and plotted. To eliminate the effects of sample scattering or refractive-indexinduced spherical aberration on the measured SHG signals, we acquire the SHG images only at the surface of the tendon specimen.

Shown in Fig. 1 are the rat tail tendon SHG images (along with histological images) acquired at six temperatures of $25^{\circ} \mathrm{C}, 40^{\circ} \mathrm{C}, 52^{\circ} \mathrm{C}, 55^{\circ} \mathrm{C}, 57^{\circ} \mathrm{C}$, and $60^{\circ} \mathrm{C}$. The thermal dependence of the SHG signal over the entire temperature range is shown in Fig. 2. A qualitative examination of Figs. 1 and 2 shows several interesting results. First, compared with lowertemperature results, the SHG images at $52^{\circ} \mathrm{C}$ and $55^{\circ} \mathrm{C}$ indicate that collagen fibers tend to demonstrate a greater degree of curvature with increasing temperature. Furthermore, although the SHG sig-

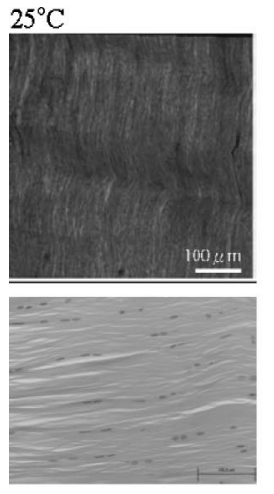

$40^{\circ} \mathrm{C}$
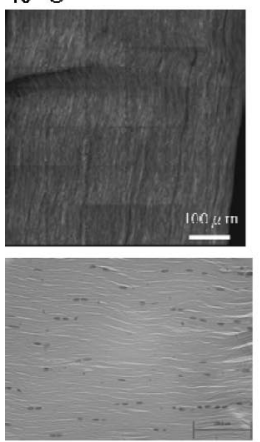

$52{ }^{\circ} \mathrm{C}$

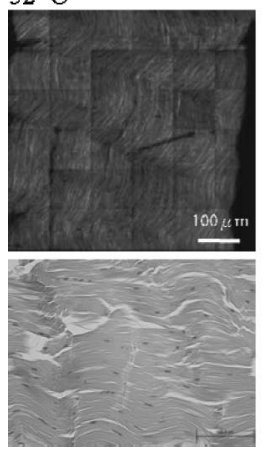

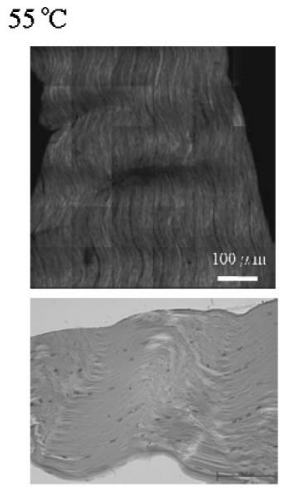

$57^{\circ} \mathrm{C}$

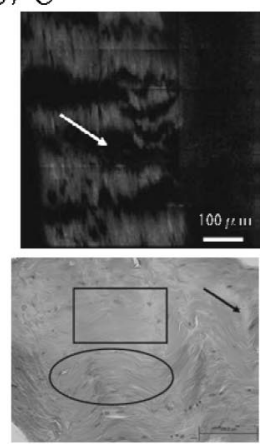

$60^{\circ} \mathrm{C}$

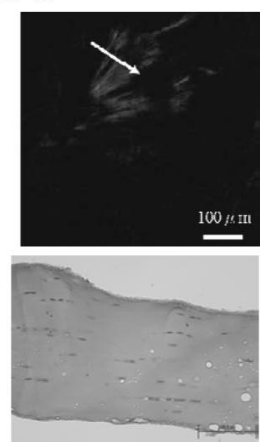

Fig. 1. SHG images $(390 \mathrm{~nm})$ of rat tail tendon treated for $10 \mathrm{~min}$ at different temperatures. Disruption of collagen structures are indicated by arrows. Histological images are shown for comparison. 


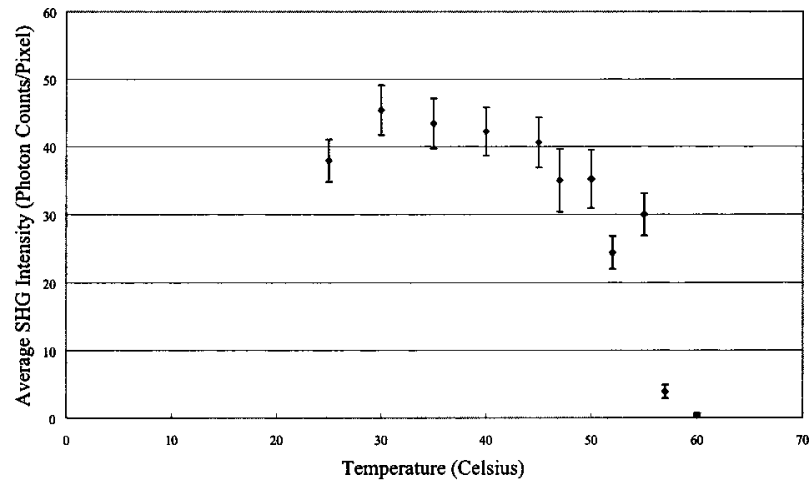

Fig. 2. Dependence of the SHG signal $(390 \mathrm{~nm})$ of rat tail tendon at different temperatures.

nals start to sharply decrease at $45^{\circ} \mathrm{C}$, breakages in collagen fibers are not evident until approximately $57^{\circ} \mathrm{C}$ (indicated by arrows). This observation, together with the decrease in the SHG signal, indicates that the structure lacking inversion symmetry responsible for the collagen SHG signal has been disrupted. At $57^{\circ} \mathrm{C}$ our SHG image shows the coexistence of two types of region with different structural organization. The SHG image still shows the existence of collagen fibers; however, there are regions within the collagen fibers in which the SHG signal is absent. This observation is supported by a histological image in which the fibrous and molten regions are indicated by elliptical and rectangular regions, respectively. At $60^{\circ} \mathrm{C}$, collagen denaturation is more complete as a further decrease of the SHG signal is correlated to a further disruption of the fibrous structures. Note that in our approach the large-area SHG scan is critical in identifying the changes to collagen fibers. In localized microscopy, features such as fiber breakage and collagen denaturation may not be easily identified.

Our results suggest that SHG intensity and images need to be combined to assess the overall changes to thermally treated collagen specimens. In addition, since the thermal response of each type of collagen tissue may be different, therapeutic procedures using thermal effects should be performed to determine the threshold damage level to collagen.
In conclusion, SHG microscopy of thermally treated rat tail tendon has shown that, despite a decrease in SHG signal at the earlier temperature of $45^{\circ} \mathrm{C}$, breakages to collagen fibers are not evident until $57^{\circ} \mathrm{C}$. At increasing temperatures, SHG images demonstrate further disruption in the collagen structure. Our results show that SHG imaging is an effective method for fully characterizing the thermal effects of collagen fibers and may be developed into an effective imaging technique for in vivo biomedical applications.

This work was supported by the National Research Programs for Genomic Medicine, Taiwan (grants NSC 92-2112-M-002-018 and NSC 92-3112-B002-048). S.-H. Jee's e-mail address is shiouhwa@ha.mc.ntu.edu.tw; C.-Y. Dong's is cydong@phys.ntu.edu.tw.

\section{References}

1. W. Denk, J. H. Strickler, and W. W. Webb, Science 248, 73 (1990).

2. P. T. C. So, C. Y. Dong, B. R. Masters, and K. M. Berland, Annu. Rev. Biomed. Eng. 2, 399 (2000).

3. A. Zoumi, A. Yeh, and B. J. Tromberg, Proc. Natl. Acad. Sci. USA 99, 11014 (2002).

4. P. J. Campagnola and L. M. Loew, Nat. Biotechnol. 21, 1356 (2003).

5. T. R. Lyons, P. L. Griffith, F. H. Savoie, and L. D. Field, Arthroscopy 17, 25 (2001).

6. M. B. McDonald, P. S. Hersh, E. E. Manche, R. K. Maloney, J. Davidorf, M. Sabry, and the Conductive Keratoplasty United States Investigators Group, Ophthalmology 109, 1978 (2002).

7. R. E. Fitzpatrick, M. P. Goldman, N. M. Satur, and W. D. Tope, Arch. Dermatol. 132, 395 (1996).

8. T. Theodossiou, G. S. Rapti, V. Hovhannisyan, E. Georgiou, K. Politopoulos, and D. Yova, Lasers Med. Sci. 17, 34 (2002).

9. B. M. Kim, J. Eichler, K. M. Reiser, A. M. Rubenchik, and L. B. Da Silva, Lasers Surg. Med. 27, 329 (2000).

10. A. T. Yeh, B. Kao, W. G. Jung, Z. Chen, J. S. Stuart, and B. J. Tromberg, J. Biomed. Opt. 9, 248 (2004).

11. Y. Sun, J. W. Su, W. Lo, S. J. Lin, S. H. Jee, and C. Y. Dong, Opt. Express 11, 3377 (2003), http:// www.opticsexpress.org. 